\title{
Impact of the solar magnetic cycle on a protoplanetary disk
}

\author{
Andrey G. Tlatov \\ Kislovodsk Mountain Station, The Central Astronomical Observatory of RAS at Pulkovo, \\ 357700, Box-145, Gagarina st., 100, Kislovodsk, Russia \\ email: tlatov@mail.ru
}

\begin{abstract}
We consider the influence of the periodic magnetic field of the Sun on the protoplanetary disk. Solar magnetic cycle may create a special orbit, which were formed main planets of the solar system. In orbits on which magnetic field accumulation occurs most effectively, there is a substance replacement. The Keplerian orbit with the period close to the period of solar magnetic cycle $T \sim T_{M}$ is most unstable for material accumulation. Two most close orbits where there is an accumulation of substance have periods $T=1 / 2 T_{M}$ and $T=5 / 4 T_{M}$. These orbits on are close to orbits of Jupiter and Saturn. Other planets were probably formed under influence of gravitation of Jupiter, Saturn and solar magnetic cycle. Perhaps, the effect of periodic magnetic field can explain the Titius-Bode rule.
\end{abstract}

Keywords. planetary systems: formation,protoplanetary disks; Sun: evolution

Magnetic fields likely play a key role in the dynamics and evolution of protoplanetary disks. They have the potential to efficiently transport angular momentum. Various rotation rates of central body and external disk resulted in increase an azimuthal component of magnetic field, increase angular momentum of protoplanetary disk and delay of central body rotation. Hardly tense magnetic spiral puts outward pressure upon gas. Thus gas goes on a spiral outside and planetosimales inside (Hoyle, 1960,Safronov \& Ruzmaikina, 1985).

Let's consider a case if time of interaction of variable magnetic field protoplanet nebulae occurs to plasma for close to solar magnetic cycle period. It is possible, if the residual magnetic field perceived by plasma, will be kept for a commensurable time interval. The dissipation time of magnetic field in plasma with small magnetic numbers Reynolds is determined by diffusion equation $\partial B / \partial t=\eta \Delta B$, where $\eta$ - magnetic diffusion coefficient which means, that variations of magnetic field with characteristic scale $l_{0}$ disappear for characteristic diffusion time: $\tau_{d}=l_{0}^{2} / \eta$. For in partially ionization plasmas $\tau_{d}=$ $3.7 \cdot 10^{2} \cdot l_{0}{ }^{2} \cdot T^{-1 / 2} \cdot n_{e} / n_{n}$. For the characteristic size protoplanetary disk $10^{12} \mathrm{~m}, \sim 10^{3}$ $K^{o}$ and ionization level $10^{-12}$ dissipation time of magnetic field is about 100 years. Thus, the magnetic field can be kept and, under certain conditions, to grow on periods of same solar magnetic cycles. It can result in effective influence of magnetic field to nebulae plasma.

Let's assume that the solar magnetic cycle existed at the earliest stages of existence of solar system when planets have not been forming yet. The period of solar magnetic cycle, probably, differed from present time, but during evolution, period of magnetic cycle changed also. Let's consider interaction mechanisms of variable magnetic field with rotary protoplanets nebulae. Let's apply some simplifying assumptions. We shall take into account that during solar magnetic cycle there is change of magnetic polarity of each solar hemisphere. Also we shall notice that inclination angle $D$ of the solar spin axis 
is not strictly perpendicular ecliptic planes and slopes for the majority planets makes $D \sim 5-7^{\circ}$.

In this case, in one half of ecliptic plane radial magnetic lines have one direction in and other half about an opposite direction. In these parts of ecliptic plane the Sun is visible under different angel $D$. During the magnetic cycle the direction of magnetic field half-planes will vary to opposite. Thus, the rotaries around the Sun plasma, hits in the magnetic field of various polarities (Tlatov, 2000). On major distances from the Sun force lines of magnetic field have preferentially radial direction $B_{r}$. At interaction with the gyred protoplanet disk from radial magnetic field can appear an azimuth component of magnetic field $B$. The magnitude $B$ can be appreciated as $B_{\varphi} \sim R e_{m} \cdot B_{r}$ in case of constant magnetic fields $B_{r}$ (Safronov \& Ruzmaikina, 1985), where $R e_{m}$-magnetic Reynolds number. If change happen slowly it is possible to accept, that plasma and magnetic field are in magnetostatic equilibrium. Pressure of plasma $p$ and components of a magnetic field and $B_{\varphi}, B_{r}$ also are coupled by relation:

$$
0=\partial P / \partial R+\partial / \partial R\left(B_{r}^{2}+B_{\varphi}^{2}\right) / 2 \mu+B_{\varphi}^{2} / \mu R-\rho \partial \Phi / \partial R
$$

where $P$-pressure, $\Phi$-gravitational potential, a $\rho$-densities. From this equation it is visible, that growth of magnetic intensity results in pushing out of substance. In case of constant magnetic field force of pushing out grows to the disk center. At a variable magnetic field the modification of a component of magnetic field $B_{\varphi}$, is described by the equation:

$$
\partial B_{\varphi} / \partial t=\eta \nabla^{2} B_{\varphi}+R \cdot B_{r} \partial \omega / \partial R,
$$

where $\eta$-coefficient of magnetic diffusion, $R$-distance from a spin axis, a $\omega$-angular velocity, $B_{r}$-magnitude of radial magnetic field. The first item characterizes a temp of a dissipation of magnetic field, second - temp of transformation of radial component of magnetic field in poloidal magnetic field. In case of solar magnetic cycle component $B_{r}$ is a variable. $B_{r}$ depends from a phase of solar cycle and an angular position of plasma region. We can accept, that magnitude $B_{r}$ is proportional $B_{r} \sim B_{r 0} \cdot \sin (\theta) \cdot \cos \left(\omega_{m} \cdot t\right)$, where $\omega_{m}=2 / T_{m}, T_{m}$-period of magnetic cycle $B_{r 0}$-intensity of dipole magnetic field near to the Sun, $\theta$ - angle between of part disk and direction on centre of the Sun. Being rotated on a Keplerian orbit regions of a disk change the relative position concerning solar equator, hence the angle $\theta$ will vary, i.e. $\theta=D \cdot \sin (\omega t+\psi)$, where a $\psi$-phase describing an angular position of a region, $\omega$ - Keplerian angular velocity of a disk. Thus $B_{r} \sim B_{r 0} \cdot \cos \left(\omega_{m} \cdot t\right) \cdot D \cdot \sin (\omega t+\psi)$. This relation reflects a change of magnetic field during magnetic cycle and rotation of protoplanet disk around of the Sun. For determination of component $B_{\varphi}$ it is necessary to carry out integration the equation (2). Let's estimate the first term in a right part of the equation (2) time Parker's instabilities (Tout \& Pringle, 1992). Diffusion transposition happens in basic through side areas protoplanet disk, we can suppose, that the equation (2) can be noted as:

$$
\partial B_{\varphi} / \partial t=B_{\varphi} / \tau_{p}+B_{r 0} \cdot \cos \left(\omega_{m} \cdot t\right) \cdot D \cdot \sin (\omega t+\psi)
$$

where $\tau_{p^{-}}$a temporal scale for Parkers instabilities $\tau_{p}=2,2 \cdot h_{d} / v_{A}$, where $h_{d}$-a gravitational scale of height, $v_{A}$ - aplfven velocity for $B_{\varphi}$ components of a magnetic field, $\psi$ a phase describing a position of a element, $f(r)$ - function of distance from central body. The scale of height $h_{d}$ can be estimated from an isothermal sound velocity and a velocity of rotation of a disk $h_{d}=c_{s} / \Omega$.

From the equation (3) it is visible, that the magnetic field differently influences on protoplanet substance depending on distance from the central body. The period of Keplerian circulation $T=T_{m}$ there is the most effective accumulation of magnetic field. In this orbit there is a linear growth of magnetic field and magnetic pressure. Concentration 


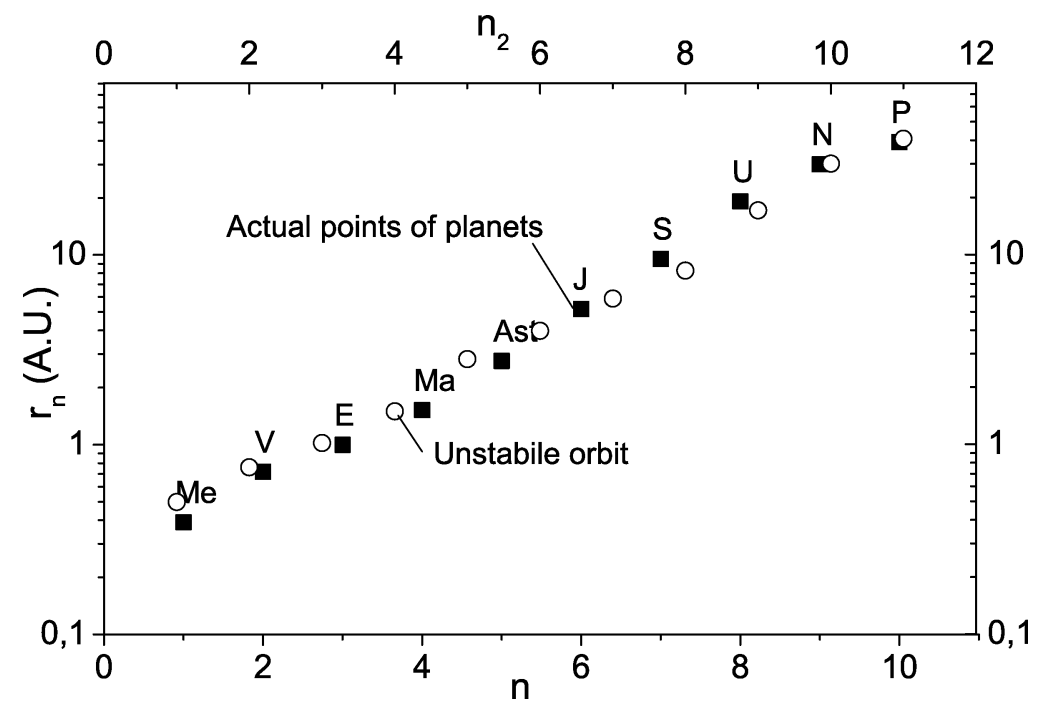

Figure 1. Actual points of planet of solar system expressed in Astronomical Units (squares) and distribution of orbits with unstable orbits in relation to accumulation of a magnetic field (circle).

of particles nebulae thus should decrease what to provide a magnetogasdynamic equilibrium (1). Thus, the substance will be aimed to abandon an orbit with period of rotation $T=T_{m}$. Also local maxima exist at periods $T=1 / 3 \cdot T_{m}$ and $T=3 \cdot T_{m}$ and others (Fig. 1). But it is possible to note, that the dissipative term restricts time of magnetic field accumulation. Thus, the substance will accumulation there where the demagnetization of plasma disk happens most fast. Let's consider an orbit with period $T=T_{m} / 2$. For this time a complete reversal the solar magnetic dipole will replace the sign on opposite and the element of plasma disk will be acted with magnetic field of opposite polarity. It means, that the second member in a right member of the equation (3) will convert in a zero in time equal to a reversal period $T=T_{m}$. Residual magnetic fields of opposite polarity will cancel each other. The orbit with phase $T=T_{m} / 2$ is preferable to substance accumulation. Now the period of magnetic cycle is about $\sim 22$ years. Hence, the orbit in which, forces of magnetic ejection on a plasma ring were minimal, was an orbit with the period about 11 years, i.e. an orbit close to Jupiter orbit. If, on the contrary, to accept, that a substance period which the Jupiter was formed corresponded to of half period of a magnetic cycle the full period was equal $T_{m} \sim 11.86 \cdot 2=23.72$ year.

\section{References}

Cameron, A. G. W. 1961, Icarus, 1, 13.

Hoyle, F. I. 1960, QJRAS, 1, 28.

Safronov, V. S. \& Ruzmaikina, T. V. 1985, in: Protostars and planets, (Tucson, AZ, University of Arizona Press), 59.

Tout, C. \& Pringle, J. E. 1992, MNRAS, 259, 604.

Tlatov, A. G. 2000, in proc. conf. "Solar in maximum activity and solar-star anolg.", ( ed. A. V. Stepanov \& S. Peterburg), 71. 